\title{
Extraction of Miao Embroidery Culture Factors Based on Perception Analysis
}

\author{
Zhou Xuan ${ }^{1}$, Zhang Jianmin* \\ College of Mechanical Engineering, Guizhou University \\ ${ }^{1}$ Graduate Student of Industrial Design, Guizhou University
}

\begin{abstract}
Through the way of creating model, this paper deeply explores and analyses the extraction of Miao embroidery cultural factors, endows traditional embroidery with new vitality, further enriches the cultural connotation of products, inherits the fine Miao embroidery culture and endows it with new vitality, promotes it to be further developed on the basis of adapting to the development environment of the new era, and better meets the diversification and individualization of various users. Firstly, this paper collects data from various channels, collects appropriate morphological samples according to relevant standards or requirements after integrating them scientifically and reasonably, chooses the method of semantic difference which is widely used at present, and compiles a five-point psychological scale which is reliable, reasonable, standardized and effective. At the same time, it discusses it comprehensively and deeply through the classical analytic hierarchy process. Various factors affecting the vocabulary of stylistic features and their importance are ranked to determine the most significant and prominent cultural factors. The main purpose of this paper is to combine the two different fields of culture and design rationally, so as to promote the further enrichment of product culture on the basis of inheriting and developing traditional culture.
\end{abstract}

\section{Introduction}

With the rapid development of the national economy, the quality of life of the general public has been significantly improved compared with before, and people's material needs have been well met. After that, more demands have been put forward for spiritual culture. To fully meet the cultural needs of the general public, we need to dig deeply into traditional culture and at the same time base ourselves on the development of the times to endow it with new meanings, and achieve a scientific and reasonable transformation of reality. Whether creative or design, they are all developed from culture, but they all transcend culture. Taking scientific and reasonable measures to excavate and innovate traditional culture helps to cater to people's experience and needs. The solid foundation of the long-standing traditional culture can further enrich the meaning of design and give more value to creativity. Combining traditional culture with creative design in a clever and reasonable way can not only fully reflect the connotation of product design, but also help to enhance the competitive advantage of products. It can be said that creative design has put on a layer of "clothing yarn" to meet the needs of the development of the times, which makes the traditional culture more practical. The purpose of this paper is to promote the effective integration between culture and design, further enrich and expand the connotation of products, and realize the effective inheritance and development of traditional culture. On the premise of carrying out morphological perception analysis, this paper will focus on the traditional patterns of Miao embroidery, comprehensively and thoroughly study their morphological composition, clarify their connotative characteristics, and scientifically and reasonably determine their morphological and sensory characteristics. Including various factors of sex, from which comb and summarize the characteristics of cultural factors. ${ }^{[1]}$

\section{Analysis of Research Status}

As a symbolic language with distinctive features, distinct and easy to understand in product design, design genes not only have strong identifiability, but also can be effectively inherited, which is helpful to enhance people's understanding of products. It has been actively applied in visual elements, showing unique characteristics, and well reflects the sustainability and consistency of a company or product. Many powerful, well-known and large-scale companies in China have launched a comprehensive and in-depth discussion and analysis of product design genes in order to enhance the competitiveness of the company while further enhancing the product image recognition, such as Unicom Company. However, compared with developed countries, China's research on cultural gene extraction has just begun. Among them, Yu Hui and other scholars focus on Banpo pottery culture, and start with a comprehensive and in-depth analysis of gene extraction 
and specific methods of cultural design from the aspects of pattern, shape and so on. In addition, from the perspective of perception image, Chen Hongyuan and other scholars have carried out in-depth and meticulous research on automobile shape characteristics, and made further analysis of the results obtained, thus obtaining standardized, accurate, objective and reasonable design data, which lays an important foundation for later design application. ${ }^{[2]}$

Effective combination of traditional culture and creative design, through scientific and reasonable methods to select the traditional culture factors with strong typicality, focus on exploring various design factors with practical value, highlighting the in-depth integration of products and culture. In this regard, this paper first gives a comprehensive and detailed description of the current widely used methods of extracting various factors, and then constructs a rigorous, perfect, scientific and reasonable model of extracting cultural factors on the premise of image analysis.

\section{Construction of Research Model}

The main role of cultural factors is to disseminate knowledge. To achieve this goal, the first problem to be addressed is to extract appropriate cultural factors accurately and efficiently. The most conventional method is to face traditional culture, extract cultural factors from the perspective of pattern and color, and then reconstruct them according to specific norms and requirements. Specifically, we need to extract cultural factors efficiently and accurately on the basis of morphological perception analysis. After preliminary integration, we need to further decompose them, and then dig cultural factors in depth from the following two aspects. On the one hand, perceptual analysis needs to compile a rigorous, reasonable, standardized and effective perceptual evaluation table. Secondly, design authorities are invited to give perceptual evaluation to get accurate and objective descriptive vocabulary of morphological style. On the other hand, feature analysis refers to the scientific and rational definition of morphological style vocabulary by combining connotation and visualization. The most representative cultural factors are determined by the way of assigning the relevant characteristic indicators. The relevant models can be referred to the following figure.

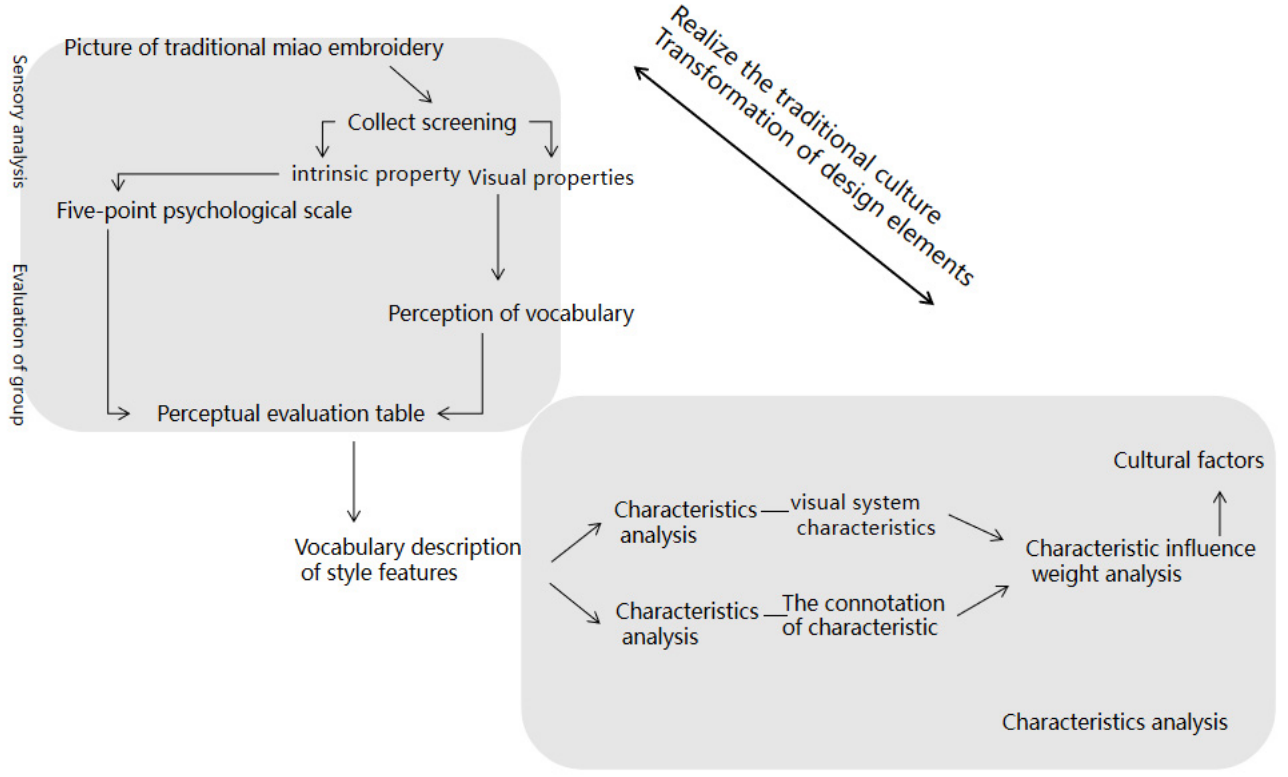

Fig.1 traditional cultural factor design factor extraction model based on perception analysis

\section{Key techniques of design culture factors extraction}

\subsection{Perception Analysis}

This paper focuses on the classical traditional Miao embroidery patterns and carries out a comprehensive and in-depth study. The first step is to collect samples through various ways and feasible means, to comprehensively consult the relevant literature and materials of this research topic, to screen out the patterns that meet the requirements, and to extract reasonable samples after removing the background; the second step is to collect emotional vocabulary, which mostly comes from pairs of pairs vocabulary. The main purpose of perceptual interpretation of embroidery patterns is to fully and accurately grasp the user's understanding and perception of such patterns, scientifically and reasonably classify the acquired vocabulary according to specific criteria, invite design students and industrial designers to score, select words with similar meanings, and create a large-scale antonym vocabulary group; the third step is to use the current application. A comprehensive and in-depth 
quantitative analysis is carried out by a relatively wide range of semantic difference methods. The rigorous, standardized, scientific and rational Likert five-point psychological scale is used to invite potential users to conduct perceptual evaluation of the selected sample contours. According to the perceptual characteristics of the curve shape, the change order of the semantic level is - 1 (very), - 0.5 (general), 0 (equal), 0.5 (general) and 1 (very). The antonyms of each sample are integrated and displayed in the figure 2 .

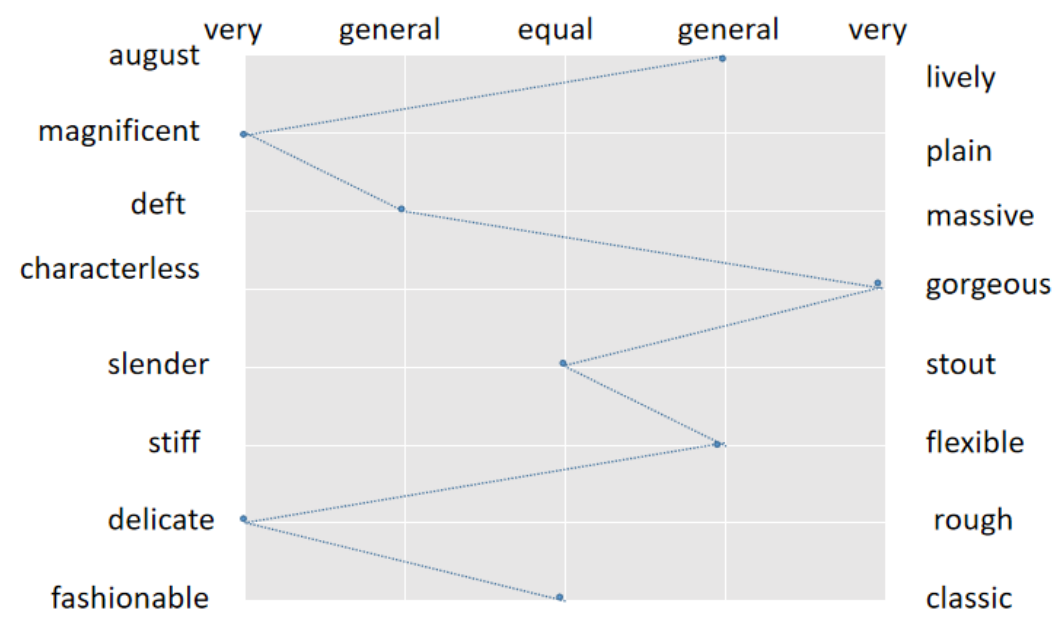

Fig. 2 Vocabulary description of style features

\subsection{Target Object Hierarchy Analysis}

In the study of design factors, we should start from the macro point of view, and then gradually refine the screening. Because the existence form of design factors is not clear, it lacks of operability in reality. The introduction and application of analytic hierarchy process (AHP) provides a new way to solve this problem. Some Western scholars not only use analytic hierarchy process, but also use factor analysis to analyze such problems. Under the guidance and assistance of analytic hierarchy process (AHP), people can subdivide macro-objects with strong uncertainty into several design feature requirements. After comparative analysis, the relative importance of each element is clarified. Several higher score of design factors are defined as important design factors of the analysed object.

\subsection{Traditional culture characteristic design factors extraction model}

The main function of design factors is to help people identify specific design objects more accurately and efficiently, which means that design factors themselves contain specific cultural connotations which can effectively disseminate culture. The knowledge mentioned here involves many aspects, such as corporate culture, regional culture and so on. In order to achieve the above goals, the first problem to be addressed is to ensure that people can form a scientific and rational understanding of design factors. Generally speaking, design factors are extracted mainly by design authorities or experts combined with personal knowledge and judgment. It is Easy to cause problems. The extraction of design factors and user perception characteristics are combined skillfully and reasonably to give full play the role of users, thereby further enhancing the recognition of design factors. Details of the extraction steps can be found in the following figure.

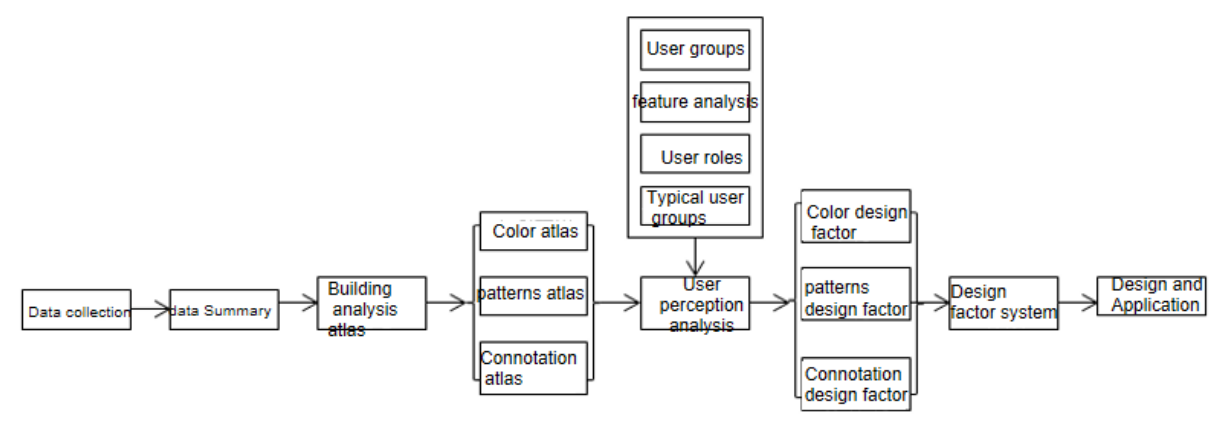

Fig. 3 Extraction Model of Design Factor of Traditional Culture Based on User Perception 


\subsection{Construction of analytical traditional culture patterns}

Analytical patterns mainly refers to the classification of samples with little difference in attributes according to specific rules or requirements. A comprehensive and indepth study of the sample patterns can help people to identify the common characteristics of various samples efficiently and accurately, so as to achieve the effective extraction of characteristic factors. Figure 4 is the traditional Miao embroidery analysis patterns.
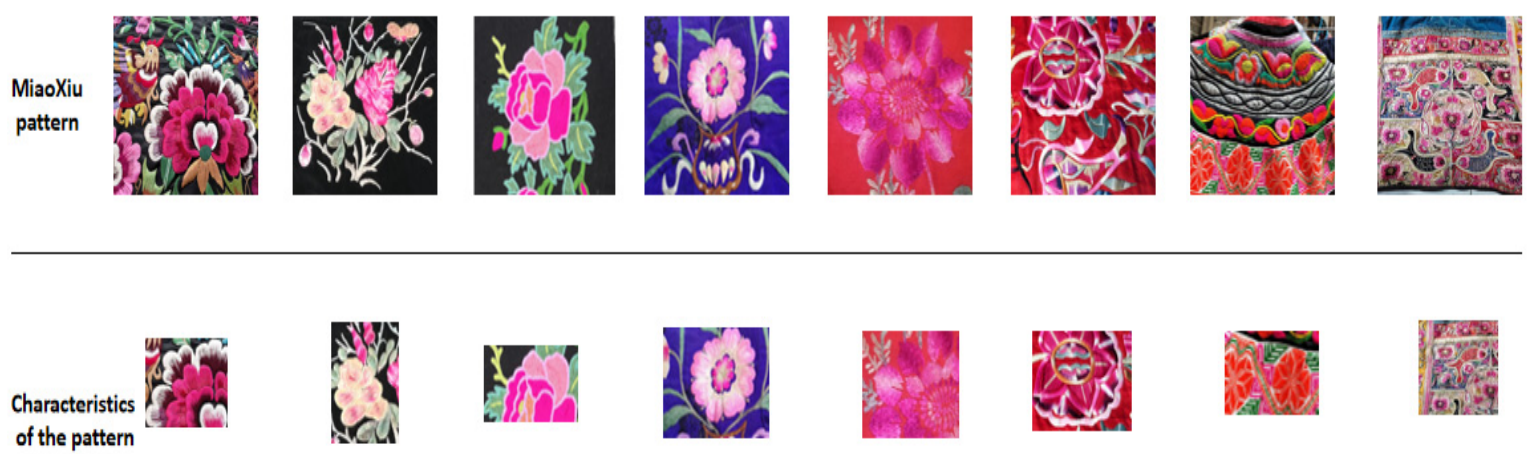

Characteristics
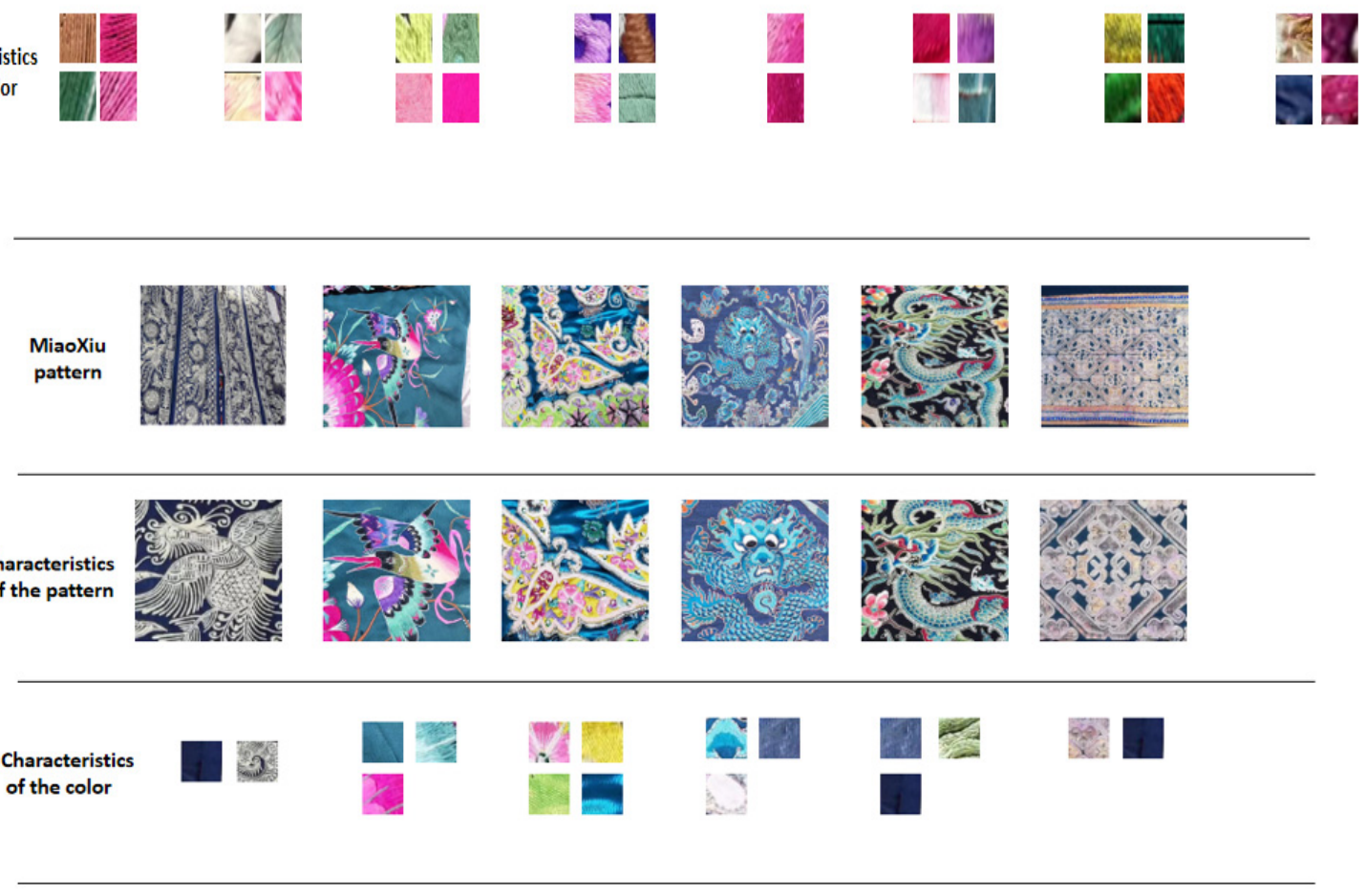

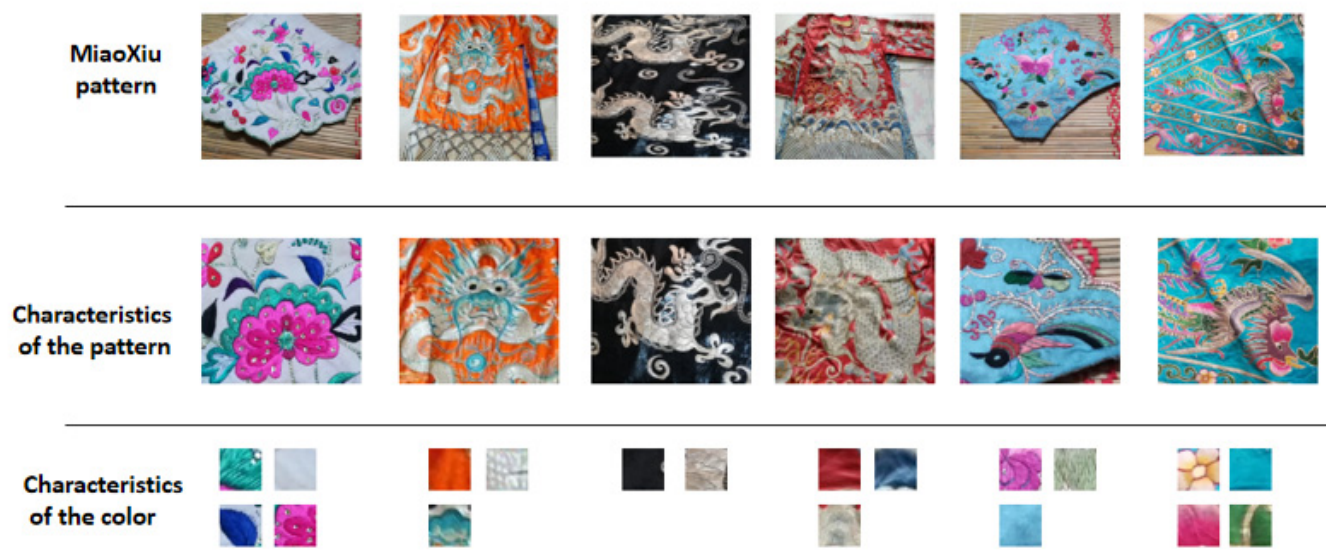

Fig. 4 Analysis Atlas of Traditional Miao Embroidery

\subsection{Construction of Analytic Hierarchy Process Matrix}

Through comparative analysis of various design features obtained by decomposition, a rigorous, reasonable and standardized scientific judgment matrix is created.

$$
A=\left(\begin{array}{cccc}
a_{11} & a_{12} & a_{1 j} & a_{1 n} \\
a_{21} & a_{22} & a_{2 j} & a_{2 n} \\
a_{11} & a_{i 2} & a_{i j} & a_{i n} \\
a_{n 1} & a_{n 2} & a_{n j} & a_{n n}
\end{array}\right)
$$

Where $\mathrm{a}_{\mathrm{ij}}$ is the importance of the $\mathrm{i}$ design factor to the $\mathrm{j}$ design factor; $\mathrm{n}$ is the total number of design factors. The value of $\mathrm{a}_{\mathrm{ij}}$ can be determined by the representative user through the currently widely used seven pole scale method (as shown in Table 1). In view of people's perceptual perception and understanding of things, a standard, reasonable, rigorous and scientific perceptual evaluation table is designed based on the reference graph, and the value of the result is taken. If the importance is the same, the value is 1 , if the importance is special. Don't be high. The value should be.

Tab.1 Perceptuale valuation table

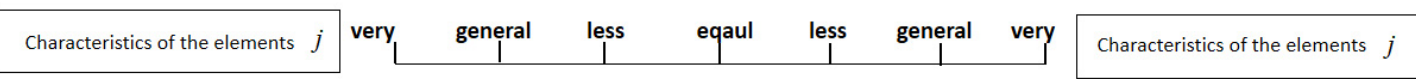

The maximum characteristic root of judgment matrix $\mathrm{A}$ is $\lambda_{\max }$, assuming that the relative importance of each design feature forms the vector $\mathrm{K}$, which is defined as $\mathrm{K}=(\mathrm{k} 1, \mathrm{k} 2, \ldots \mathrm{kn})$, from which $A \bullet k=\lambda \max \bullet k$ can be deduced. After calculating the equation, we can get:

$$
k_{i}=1 / n \cdot \sum_{j-1}^{n} \overline{a_{i j}}
$$

\subsubsection{Calculating design features}

If a total of $\mathrm{X}$ users are included in the user group, all the perceptual results provided by $\mathrm{X}$ users should be considered comprehensively and deeply when calculating and analyzing, so as to obtain the comprehensive importance of the feature factors:

$$
K_{i=\frac{1}{X}}^{\prime}\left(k_{i}^{(1)}+k_{i}^{(2)}+\cdots+k_{i}^{(x)}\right)
$$

Among them, $\mathrm{X}$ represents the total number of typical users; the higher the value of $k_{i}^{\prime}$, that is, the higher the importance of the first $\mathrm{i}$ element, and the higher the probability that this element is defined as a characteristic factor.

\subsubsection{Reconstructing Analysis Atlas}

The traditional analysis Atlas of Miao nationality in Figure 4 can easily find common design factors, such as patterns, connotations and so on. In order to facilitate user perception and evaluation, the design factors obtained should be scientifically and reasonably coded according to specific requirements or standards, Group a ( ) is pattern design factor, group b ( ) is color design factor and group $\mathrm{c}(\mathrm{c} 1, \mathrm{c} 2, \mathrm{c} 3)$ is connotation or story design factor. Thereby this article creates a complete and rigorous user perception evaluation atlas, which can be referred to the table 2 . 
Tab 2. User perception evaluation Atlas of Miao embroidery design factors

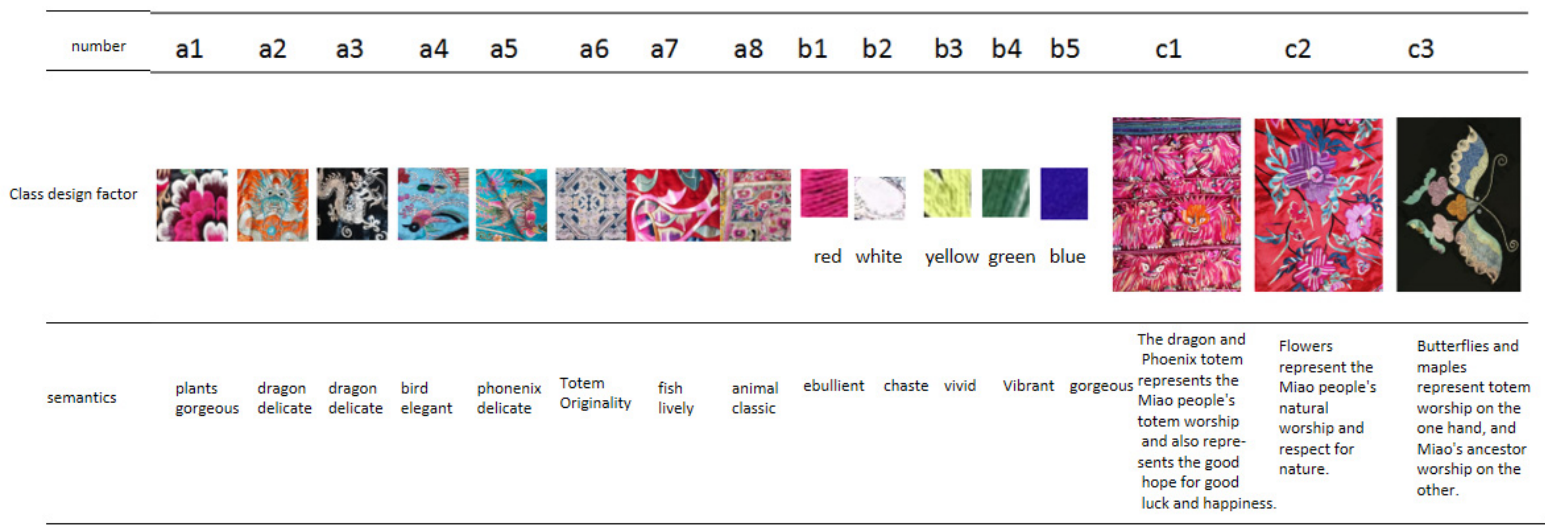

\subsubsection{Design Culture Factors Establishment}

Based on the investigation of perceptual intention, the typical design factors of Miao embroidery culture are determined, which can be referred to in the table 3 .

Tab.3 Statistics of design factors with large $k^{\prime}{ }_{i}$ value

\begin{tabular}{rrrrrrr}
\hline $\begin{array}{l}\text { Design factor } \\
\text { a1 }\end{array}$ & a5 & a6 & a7 & a8 \\
$k^{\prime} i$ & 0.0083 & 0.0069 & 0.0121 & 0.0196 & 0.0057 \\
\hline Design factor & b1 & b3 & b5 & c1 & c2 \\
$k^{\prime} i$ & 0.0258 & 0.0182 & 0.0182 & 0.0519 & 0.0427
\end{tabular}

From Table 3, we can see that the design factors a7, b1 and $\mathrm{c} 1$ have the highest scores in the three group: a, b and c. From this, we can see that the fish pattern (a1), and color red (b1) and dragon and Phoenix totem (c1) contain the most connotative factors which can represent Miao embroidery in the eyes of most consumers. From this, we can get the key design factor of three design schemes; $a, b$ and $\mathrm{c}$.

\subsection{Design Schemes Generation}

Under the guidance of modern design concepts, a series of tourism creative products with traditional cultural connotations and in line with the development needs of the times have been developed through scientific and rational innovation of the original design factors through the current widely used methods.According to the establishment of design factors, we can get two directions of design methods. On the one hand, we can simply use patterns to express them in different ways in various products; on the other hand, we can combine embroidery with modern products to apply embroidery to the design of different products; from this, we can get Mark 6, which is a combination of class a design factors and modern design products; on the other hand, we can get 7 eyes. Cover is a combination of class B design factor and modern design, and the luggage box in Figure 8 is a combination of class $\mathrm{C}$ connotation culture factor and modern design.
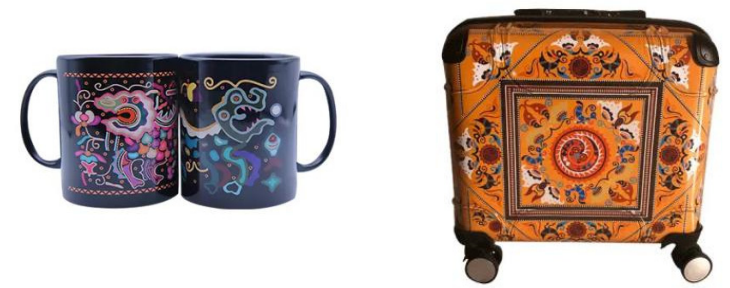

Fig. 5 mug cup

Fig.6 blind mask

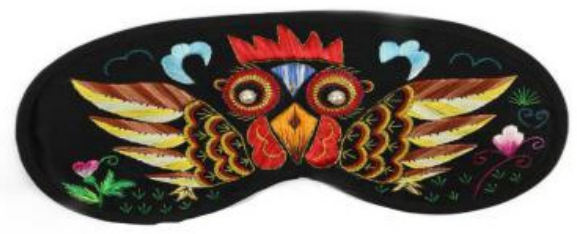

Fig.7 Luggage case

\section{Conclusion}

Traditional cultural creative product design plays an important role in our cultural industry. Simply speaking, it is an organic combination of multi-disciplinary knowledge such as culture, art, technology and so on. The rational extraction and practical application of its characteristic design factors can further enrich the connotation of the design object, promote it to display more fully the unique charm of traditional culture, so as to make it more recognized by more people.Know and understand the mysterious and ancient traditional culture of ethnic minorities.In this regard, this paper takes user research as the starting point, through a combination of various methods to determine cultural design factors efficiently and reasonably, hoping that this paper can achieve a clever integration between traditional culture and modern design to provide a new way of thinking. 


\section{Author}

Zhang Jianmin (1976-), female, Xingren, Guizhou Province, Associate Professor of Industrial Design, Guizhou University, Master \& apos; s supervisor.

\section{Reference}

1. KARJALAINEN T M.It Looks Like a Toyota: Educational Approaches to Designing for Visual Brand Recognition [J] International Journal of Design, 2007 (1): 67-81.

2. KARJALAINEN T M. When is a Car Like a Drink Metaphoas a Means to Distilling Brand and Product Identity [J] Design Management Journal, 2001, 12 (1): 66-71.

3. JAY P M. Speaking the Buick Language: Capturing, Understanding, and Exploring Brand Identity with Shape Grammars [J].Design Studies, 2004, 25 (1): 129.

4. Luo Shi jian, Zhu Shang shang, Feng Cheng. Product family design DNA for industrial design [J]. Journal of Mechanical Engineering, 2008 (7): 123-128

5. LUO Shi-jian, ZHU Shang-shang, FENG Cheng. ProducFamily DNA in Industrial Design [J]. Chinese Journal of Mechanical Engineering, 2008 (7): 123128.

6. Gou Bing chen, Yu Hui. Applied Research on Extraction and Design of Cultural Genes of Banpo Pottery [J]. Journal of Northwest Polytechnic University (Social Science Edition), 2011 (4): 66-69.

7. GOU Bing-chen, YU Hui. Banpo Painted Pottery Culture Gene Extraction and Design Applications [J].Journal of Northern Western Polytechnical University (Social Sciences), 2011 (4): 66-69.

8. Yang Xiao yan, Wang Wei wei. Design and Research of Culture-oriented Urban Sign System [J]. Packaging Engineering, 2010, 31 (18): 77-80.

9. YANG Xiao-yan, WANG Wei-wei. Research on Urban Identification System Design Depending on Regional Culture [J]. Packaging Engineering, 2010, 31 (18): 77-80.

10. Chen Hong yuan, Zhang Yu ming. Study on the relationship between the shape and characteristics of automobile contour [J]. Journal of National Cheng Kung University, 2009 (2): 87-105.

11. CHEN Hong-yuan, ZHANG Yu-ming. Study on the Feature Relationship of Automobile Zontour [J]. Journal Design of National Cheng Kung University, 2009 (2): 87-105.

12. Wang Wei wei, Yu Sui huai. Aircraft cabin interior environment design evaluation based on Fuzzy-AHP [J]. Aviation Manufacturing Technology, 2010 (10): 80-84.

13. WANG Wei-wei, YU Sui-huai. The Evaluation of Interior Environment Design of Airplane Passenger Cabin Based on Fuzzy AHP [J]. Aeronautical Manufacturing Technology, 2010 (10): 80-84.
14. Gong Gui fen, Yinke. Study on the Evaluation Model of Packaging Scheme Based on Fuzzy Analytic Hierarchy Process [J]. Packaging Engineering, 2011, 32 (21): 64-67.

15. GONG Gui-fen, YIN Ke. Study on Assessment Model of Packaging Solutions Based on AHP Method [J]. Packaging Engineering, 2011, 32 (21): 64-67.

16. Shen Hui, Ming Xinguo. Preliminary study on product profile management technology [J]. Mechanical design and research, 2008 (2): 72-77.

17. SHEN Hui, MING Xin-guo. Research Strategy of Product Portfolio Management [J].Machine Design and Research, 2008 (2): 72-77 